\title{
Small molecule tyrosine kinase inhibitors in pancreatic cancer
}

\author{
Sachin Gupta \\ Bassel F El-Rayes \\ Department of Hematology/Oncology, \\ Karmanos Cancer Institute, Wayne \\ State University, MI, USA
}

\begin{abstract}
Pancreatic cancer has proven to be chemo-resistant, with gemcitabine being the only cytotoxic agent approved for advanced pancreatic cancer since 1996. Tyrosine kinase inhibitors represent a newer generation of chemotherapeutic agents targeting specific tumor pathways associated with carcinogenesis including cell cycle control, signal transduction, apoptosis and angiogenesis. These agents present a more selective way of treating pancreatic cancer. Erlotinib is the prototype of the tyrosine kinase inhibitors with proven efficacy in advanced pancreatic cancer and has been recently approved in that setting. Multiple other tyrosine kinase inhibitors targeting the VEGFR, PDGFR, and Src kinases are in various phases of clinical trials testing. The preliminary results of these trials have been disappointing. Current challenges in pancreatic cancer clinical trials testing include improving patient selection, identifying effective combinations, improving the predictive value of current preclinical models and better study designs. This review summarizes the present clinical development of tyrosine kinase inhibitors in pancreatic cancer and strategies for future drug development.
\end{abstract}

Keywords: pancreatic cancer, erlotinib, tyrosine kinase inhibitors

\section{Introduction}

Pancreatic cancer is the fourth leading cause of cancer deaths in the US with more than 30,000 deaths annually (Jemal et al 2007). More than $75 \%$ of patients with pancreatic cancer present with locally advanced or metastatic disease (El-Rayes and Philip 2003). Median survival remains poor and is estimated to be about 6 months for people with metastatic disease (Bramhall et al 1995). Pancreatic cancer is resistant to both conventional chemotherapy and radiation (Brand and Tempero 1998).

Gemcitabine is the only cytotoxic drug approved for advanced pancreatic cancer in the first-line setting (Burris et al 1997). In a pivotal phase III trial, gemcitabine was compared to fluorouracil (5-FU) in previously untreated pancreatic carcinoma. Gemcitabine resulted in a significant improvement in the clinical benefit score (pain control, weight gain and performance status) over 5-FU. The objective response to gemcitabine was less than $10 \%$. An improvement in median survival from 4.2 to 5.7 months was observed. The overall survival at 1 year improved from $2 \%$ to $18 \%$. Gemcitabine has become the standard of care (Regine and Abrams 2006; Oettle et al 2007) for advanced pancreatic cancer. Combination chemotherapy regimens based on the gemcitabine platform have failed to show any significant survival advantage over single agent gemcitabine (Rocha Lima et al 2004; Louvet et al 2005).

The development of agents targeting specific tumor pathways including tyrosine kinases has been viewed as a promising approach for the treatment of pancreatic cancer. The initial results of clinical trials of tyrosine kinase inhibitors in pancreatic cancer have however been disappointing.
Correspondence: Bassel El-Rayes

4100 John R Detroit, MI-4820I, USA

$\mathrm{Tel}+|3| 35768720$

Email elrayesb@karmanos.org 


\section{Tyrosine kinases in cancer Cytoplasmic TKs and receptor TKs}

Tyrosine kinases are a major subclass of protein kinases that play a pivotal role in intracellular signal transduction (Kleespies et al 2006). Tyrosine kinases can be either receptor tyrosine kinases (RTK) or non-receptor cytoplasmic tyrosine kinases (Levitzki and Gazit 1995; Levitzki 1999). All RTKs contain an extracellular ligand binding domain that is connected to the cytoplasmic catalytic domain by a transmembrane helix. RTKs are activated by various growth factor ligands such as epidermal growth factor (EGF), vascular endothelial growth factor (Levitzki 1999; Schlessinger 2000). Ligand binding induces dimerization of the cell-surface receptors resulting in autophosphorylation of the cytoplasmic domains and activation of the receptor's tyrosine kinase (Olayioye et al 2000; Schlessinger 2000). Specifically, RTKs catalyze transfer of phosphates from ATP to the hydroxyl groups of tyrosines on target proteins. Phosphorylated tyrosines serve as binding sites for a number of cytoplasmic signal-transducing molecules, activating downstream signalling pathways (Schlessinger 2000; Yarden and Sliwkowski 2001). Downstream signalling occurs mainly via the Ras/Raf/MAP kinase pathway, the phosphatidyl-inositol phosphate kinase (PIP Kinase/PI-3K)/AKT pathway, and the JAK/STAT nuclear translocation. Activation or inhibition of these pathways modulates cell cycle processes like migration, metabolism, apoptosis and survival and differentiation. RTK function can be dysregulated by multiple mechanisms including oncogene mutations leading to constitutive activation of the kinase, amplification of the RTK gene, over-production of growth factors ligands or abnormalities in the inhibitory pathways.

Cytoplasmic tyrosine kinases on the other hand do not have an extracellular receptor and are located in the cytoplasm, nucleus or plasma membrane. They consist of eight families with the major ones including SRC, JAK, ABL, FAK (Levitzki 1999).

\section{EGFR targeting in pancreatic cancer}

The Erb family consists of four related receptors: the EGFR (EGFR/HER-1/ErbB-1), ErbB-2 (HER-2/neu), ErbB-3 (HER3) and ErbB-4 (HER-4) (Olayioye et al 2000; Arteaga 2001; Yarden and Sliwkowski 2001). Enhanced signaling from these receptors due to mutation or over-expression contributes to several types of human cancers including lung, breast, colorectal, brain, head and neck, ovarian and pancreatic (Yarden and Sliwkowski 2001; Hubbard 2006). Elevated expression of the EGFR or its ligand is also associated with worse clinical outcomes in a variety of epithelial cancers including colorectal, pancreatic, head and neck, and breast cancer (Yamanaka et al 1993; Dong et al 1998; Papageorgio and Perry 2007). ErbB-1 receptor expression is detected in upto $90 \%$ of pancreatic tumors (Lemoine, Hughes et al 1992). Over-expression of EGFR signalling is believed to induce increased survival, proliferation, chemoresistance and invasiveness of pancreatic tumors (Yamanaka et al 1993).

EGFR can be targeted therapeutically by monoclonal antibodies or small molecule tyrosine kinase inhibitors (TKI). Monoclonal antibodies interact with the extracellular domain of EGFR, inhibiting EGF ligand binding and inducing receptor endocytosis. Occasionally the Fc portion of some monoclonal antibodies may induce antibody-dependent cellular cytotoxicity (ADCC), mediating tumor cell lysis. The small molecule TKIs act intracelullarly by interfering with the ATP binding site of the EGFR tyrosine kinase thereby inhibiting its trans-phosphorylation of tyrosine residues on target proteins (Ritter and Arteaga 2003). In-vitro both the monoclonal antibodies and the small molecule TKIs efficiently block the EGFR signal transduction pathways inducing tumor cell cycle arrest, potentiating apoptosis, inhibiting angiogenesis, inhibiting tumor invasion and metastasis and augmenting chemo-radiotherapy anti-tumor effects (Mendelsohn and Baselga 2006).

\section{Erlotinib}

Erlotinib (Tarceva ${ }^{\circledR}$, OSI-774) is a reversible oral ErbB-1 tyrosine kinase inhibitor approved in pancreatic cancer. Five hundred sixty-nine patients with locally advanced or metastatic pancreatic cancer were randomized to receive gemcitabine plus placebo or gemcitabine and erlotinib $100 \mathrm{mg}$ a day (Moore, Goldstein et al 2007). Primary endpoint was overall survival with secondary endpoints being progression-free survival, response rate and improvement in the quality of life. The erlotinib plus gemcitabine arm had a higher median survival of 6.2 months versus 5.9 months with a hazard ratio of $0.82(95 \% \mathrm{CI} ; 0.67-0.97, \mathrm{p}=0.034)$. One-year survival was $23 \%$ in the erlotinib plus gemcitabine arm versus $17 \%$ in the gemcitabine alone arm. In multivariate analysis significant predictors of survival were treatment with erlotinib, good performance status, locally advanced disease and normal serum albumin. The progression-free survival was also favorable in the erlotinib arm (3.75 months versus 3.55 months) with a hazard ratio of $0.76(p=0.03)$. Although there was a higher incidence of grade 1 and 2 rash 
Table I Ongoing clinical trials evaluating tyrosine kinase inhibitors in pancreatic cancer

\begin{tabular}{|c|c|c|c|}
\hline Drug & Class & Phase & Concurrent therapy \\
\hline \multicolumn{4}{|l|}{ I. Erb inhibitors } \\
\hline erlotinib & EGFR & II & RAD00I (everolimus) \\
\hline erlotinib & EGFR & II & bevacizumab, gemcitabine \\
\hline erlotinib & EGFR & II & gemcitabine, oxalipaltin \\
\hline erlotinib & EGFR & III & capecitabine, gemcitabine \\
\hline erlotinib & EGFR & III & gemcitabine, capecitabine, radiation \\
\hline erlotinib & EGFR & $\mathrm{I} / \mathrm{II}$ & bevacizumab, gemcitabine, capecitabine \\
\hline erlotinib & EGFR & $\mathrm{I} / \mathrm{II}$ & cetuximab \\
\hline erlotinib & EGFR & $1 / I I$ & gemcitabine \\
\hline erlotinib & EGFR & II & gemcitabine, capecitabine, radiation \\
\hline erlotinib & EGFR & II & bevacizumab \\
\hline erlotinib & EGFR & II & gemcitabine, panitumumab \\
\hline lapatinib & Pan Erb & II & gemcitabine \\
\hline \multicolumn{4}{|c|}{ 2. Combined Erb/VEGFR inhibitors } \\
\hline BMS-6905I4 & Pan Erb/VEGFR & I & - \\
\hline vandetanib (ZD6474) & EGFR/VEGFR & I & gemcitabine, capecitabine \\
\hline \multicolumn{4}{|l|}{ 3. VEGFR inhibitors } \\
\hline sunitinib & VEGFR & III & - \\
\hline sunitinib & VEGFR & I & gemcitabine \\
\hline PTK787/ZK222584 & VEGFR & II & - \\
\hline PTK787/ZK222584 & VEGFR & $1 / I I$ & gemcitabine \\
\hline axitinib (AG-0|3736) & VEGFR & III & gemcitabine \\
\hline brivanib (BMS-582664) & VEGFR/FGFR & II & - \\
\hline \multicolumn{4}{|c|}{ 4. CombinedVEGFR/PDGFR inhibitors } \\
\hline sorafenib & VEGFR/PDGFR & III & gemcitabine \\
\hline sorafenib & VEGFR/PDGFR & $1 / I I$ & oxalipaltin, capecitabine \\
\hline sorafenib & VEGFR/PDGFR & I & sirolimus \\
\hline \multicolumn{4}{|l|}{ 5. PDGFR inhibitors } \\
\hline Imatinib & PDGFR & II & Gemcitabine \\
\hline \multicolumn{4}{|l|}{ 6. Src kinase inhibitors } \\
\hline Dasatinib & Src kinase & II & - \\
\hline Dasatinib & Src kinase & $1 / I I$ & Gemcitabine, Cetuximab \\
\hline AZD0530 & Src kinase & $\mid / I I$ & Gemcitabine \\
\hline
\end{tabular}

and diarrhea in the erlotinib arm, there were no significant differences in grade 3 and 4 toxicities. No correlation could be found between EGFR expression by immunohistochemistry and clinical benefit from erlotinib. Based on this modest benefit, erlotinib was approved for use in combination with gemcitabine in patients with pancreatic cancer who have received no prior therapy.

\section{Single agent erlotinib in advanced pancreatic cancer}

Erlotinib as a single agent was evaluated in patients with pancreatic cancer who had failed prior chemotherapy (Epelbaum et al 2007). The primary end-point for evaluation was progression-free survival. Thirteen patients were treated with single agent erlotinib with median treatment duration being 2 months. Median time to tumor progression was 1 month. Five patients (38\%) showed clinical improvement or disease stabilization with decrease in tumor markers. Thus erlotinib seems to have activity as a single agent in pancreatic cancer but needs to be investigated further in this setting.

\section{Gefitinib}

Gefitinib (Iressa ${ }^{\circledR}$, ZD1839) is an oral small molecule TKI specifically targeting ErbB-1 receptor. Gefitinib completely abolished EGF-induced phosphorylation of EGFR/MAP 
kinase and inhibited EGF-induced cell growth and invasion in pancreatic cancer cell lines (Li et al 2004). Efficacy of gefitinib in combination with docetaxel was tested in a phase II study of metastatic pancreatic cancer patients who had failed prior gemcitabine-based chemotherapy (Blaszkowsky et al 2007). Primary endpoint of the study was CA19-9 response $(>50 \%$ decrease in tumor marker CA19-9 levels) with additional endpoints including radiologic response, toxicity, and survival. Fifteen patients were treated with gefitinib and docetaxel with good tolerance and minimal toxicities. No patient experienced a biochemical (CA19-9) response to treatment, although 9 out of 15 evaluable patients experienced stable disease. The combination was reported to be inactive in metastatic pancreatic cancer and the trial was stopped due to lack of efficacy.

\section{Lapatinib}

Lapatinib is a small molecule TKI that reversibly inhibits ErbB-1 and ErbB-2. Lapatinib had shown activity against pancreatic tumor cell lines and was tested in a phase 1 trial in advanced pancreaticobiliary cancer (Safran et al 2006). A two-stage trial using lapatinib with gemcitabine and gemcitabine/oxalipaltin combination was conducted enrolling 21 patients, 15 with pancreatic cancer and 5 with biliary cancer. Five of the 20 evaluable patients showed partial responses with dramatic responses seen in patients with diffuse liver and peritoneal metastases. The results of the trial demonstrated that lapatinib can be safely combined with gemcitabine based chemotherapy. The preliminary efficacy results were encouraging.

\section{Future strategies targeting EGFR pathways}

Although there was a statistically significant improvement in overall and median survival with the addition of erlotinib, most physicians agree that the improvement is at best modest. The negative result of the phase III trial with cetuximab (Philip et al 2007) confirms that targeting the EGFR pathway in pancreatic cancer has limited benefit. Newer approaches being evaluated in clinical trials in pancreatic cancer include:

1. Use of agents that target multiple members of the Erb family like lapatinib. The rationale is that hetero-dimerization of EGFR with other members of the Erb family may contribute to the resistance against selective EGFR inhibitors. Therefore, targeting multiple members of the Erb family may be necessary to block the growth/survival signal from the EGFR pathway.
2. Combining erlotinib with other biologic agents. Since the signalling pathway in pancreatic cancer is dysregulated at multiple levels, inhibition of only the RTK may be insufficient to alter the chemoresistant nature of pancreatic cancer. Erlotinib was studied in combination with antiangiogenic agents such as bevacizumab in advanced pancreatic cancer. In a phase III trial, 607 chemonaive patients with metastatic pancreatic adenocarcinoma were randomized to gemcitabine and erlotinib or gemcitabine, erlotinib and bevacizumab (Vervenne et al 2008). The primary endpoint was overall survival. Median overall survival was 6 months in the gemcitabine/erlotinib arm versus 7.1 months in the gemcitabine/erlotinib/bevacizumab arm (HR 0.89; 95\% CI 0.74-1.07). Median progression free survival was 3.6 months in gemcitabine/erlotinib arm versus 4.6 months in the combination arm (HR 0.73; 95\% CI 0.61-0.86; $\mathrm{p}=0.0002)$. No clinically relevant differences were observed in clinical response and Grade 3-4 adverse events between the two arms. At our institution, we are evaluating the combination of erlotinib with inhibitors of the Akt/ NF-kB.

3. Combining EGFR blockade with non-gemcitabine based cytotoxic agents such as docetaxel or ixabepilone.

4. Combining erlotinib with radiation. Erlotinib has been analyzed in a phase I study in combination with chemoradiation followed by maintenance erlotinib for locally advanced pancreatic cancer (Iannitti et al 2005).

\section{VEGF targeting in pancreatic cancer}

Tumor progression and metastasis is critically dependent upon angiogenesis (Folkman 1971; Hicklin and Ellis 2005). Angiogenesis depends on the balance of several pro and anti angiogenic factors. Various tumor cells including pancreatic cancer commonly secrete a potent pro-angiogenic factor, the vascular endothelial growth factor (VEGF). VEGF-A increases microvascular permeability, stimulates endothelial cell migration, proliferation and protects them from apoptosis (Ferrara 1999; Dvorak 2002). VEGF - A mediates its effects by interacting with its two high affinity vascular endothelial growth factor receptors (VEGFR): VEGFR-1 (Flt-1) and VEGFR-2 (KDR/Flk-1). VEGR-1 and VEGFR-2 are high affinity transmembrane tyrosine kinase receptors selectively expressed by normal vascular and over-expressed in tumor endothelium (Dvorak 2002). VEGFR-3 (Flt-4) is another VEGFR that binds to other members of the VEGF ligand family, VEGF-C and VEGF-D. VEGFR-3 is normally 
expressed in the lymphatic endothelium and is also over-expressed in tumor cells. Similar to the EGFR signalling pathways, VEGF ligands on binding to their VEGF receptors initiate phosphorylation of the receptor tyrosine kinases. This sets off a cascade of downstream signalling events resulting in the activation of phospholipase $\mathrm{C} \gamma, \mathrm{PI}-3 \mathrm{~K}$ and Ras/MAP Kinase, mediating endothelial and lymphatic cell migration and proliferation (Dvorak 2002).

Oncogene/tumor suppressor gene activation, tissue hypoxia and cytokine secretion by pancreatic cancer cells results in increased expression of VEGF and VEGFR (Cherrington et al 2000; Baker et al 2001; Dvorak 2002). Higher serum and intra-tumoral expression levels of VEGF have been associated with larger tumor size, enhanced local progression (Itakura et al 2000), earlier recurrence after surgical resection (Niedergethmann et al 2002) and an overall worse prognosis in pancreatic cancer (Ikeda et al 1999; Luo et al 2001; Buchler et al 2002). Inhibition of the VEGF pathway has also been shown to make pancreatic cell line more sensitive to the cytotoxic effects of conventional chemotherapy like gemcitabine (Bruns et al 2002). VEGF signaling pathways have been targeted for treatment by the use of anti-VEGF monoclonal antibodies, antibodies binding to VEGFR and VEGFR tyrosine kinase inhibitors.

\section{Vatalanib (PTK787/ZK222584)}

Vatalanib is a pan-VEGFR tyrosine kinase inhibitor with activity against VEGFR-1, VEGFR-2 and VEGFR-3. Vatalanib also has activity against platelet derived growth factor receptor (PDGFR) and c-kit (Wood et al 2000). After demonstrating activity in preclinical studies and orthotopic pancreatic cancer models, vatalanib has been tested in phase I settings, the preliminary results of which were recently reported (Kuo T. 2006). In this study advanced pancreatic cancer patients are being treated with vatalanib and gemcitabine. Eleven patients have been enrolled so far, with 2 patients (18\%) showing a partial response and 6 patients $(55 \%)$ with stable disease. The combination of gemcitabine and vatalanib is well-tolerated with some antitumor responses observed and accrual is continuing.

\section{Vandetanib}

Vandetanib (ZD-6474) is a TK inhibitor of VEGFRR-2, VEGFR-3, Ret and EGFR (Ciardiello et al 2003) thus inhibiting both the VEGF dependent angiogenesis and EGFR dependent tumor cell proliferation. Vandetanib has shown significant activity in preclinical models with enhanced synergism with gemcitabine (Bruns 2003; Conrad et al 2007) prompting it to be tested in clinical settings. Vandetanib is being studied in combination with gemcitabine in patients with pancreatic cancer.

\section{Axitinib (AG0 I3736)}

Axitinib (AG-013736) is a small molecule TKI with potency against pan VEGF receptors and against PDGFR- $\beta$ and c-Kit (Spano JP 2006). Axitinib in preclinical studied has been shown to be a very effective anti-angiogenesis agent (Inai et al 2004). A phase I study in solid tumors identified $5 \mathrm{mg}$ twice a day as the therapeutic dose (Rugo et al 2005). AG-013736 was subsequently studied in combination with gemcitabine as first-line therapy for advanced pancreatic cancer in a phase II trial (Spano et al 2008). One hundred and eight patients were randomized in a 2:1 ratio to receive gemcitabine and axitinib $(n=69)$ or gemcitabine alone $(\mathrm{n}=34)$. Primary endpoint was overall survival. Median overall survival was 6.9 months $(95 \%$ CI 5.3-10.1) in the combination arm versus 5.6 months (95\% CI 3.9-8.8) in the gemcitabine alone arm. Except for grade 3 fatigue, the safety profile was similar. Based on the results of this study gemcitabine and axitinib will be evaluated further in a phase III trial.

\section{PDGFR targeting in pancreatic cancer}

Platelet-derived growth factor ligands, PDGF-A and PDGF-B, exert their effects on target cells by activating the two structurally related protein tyrosine kinase receptors, PDGFR- $\alpha$ and PDGFR- $\beta$. Activation of PDGFR leads to downstream signal transduction via PI-3K, Phospholipase $\mathrm{C} \gamma$, Ras/MAPK, Src kinase and Jak/Stat kinase pathways. Aberrant synthesis of PDGF, over-expression of PDGFR and concomitant autocrine growth stimulates neoplastic conversion of PDGFR-positive cells (Heldin and Westermark 1999). PDGFR signaling has also been reported to stimulate angiogenesis and recruit pericytes which stabilize the tumor vasculature (Bergers et al 2003; Ostman 2004; Yokoi et al 2005). PDGFR signaling in the tumor raises stromal interstitial fluid pressure, inhibiting effective transvascular transport of chemotherapeutic agents into the tumor (Pietras et al 2002; Yokoi et al 2005). PDGFRs and its ligand are overexpressed in various solid tumors including glioblastoma, sarcoma, breast, colorectal carcinoma and pancreatic cancer (Ebert et al 1995; Hwang et al 2003). Ebert et al demonstrated that pancreatic tumor samples exhibited a significant increase in PDGFR- $\alpha$ and PDGFR- $\beta$ mRNA levels compared with samples of normal pancreas (Ebert et al 1995). 
Various approaches inhibiting PGFR signaling have included monoclonal PDGF receptor antibodies that block ligand binding and low molecular weight PDGFR TKIs.

\section{Imatinib}

Imatinib is a small molecule PDGFR TKI which in animal pancreatic cancer models was shown to have a limited effect on inhibiting tumor growth and prolonging survival but was very effective when combined with other chemotherapy agents like gemcitabine (Hwang et al 2003; Yokoi et al 2005). Imatinib is thought to lower PDGFR mediated tumor interstitial hypertension, promoting transvascular transport of concurrent chemotherapeutic agents, although it lacks any significant anti-tumor effect of its own (Pietras et al 2002). Imatinib was evaluated in advanced pancreatic cancer in a small clinical trial (Chen et al 2006). Twenty Six patients were randomly treated with either gemcitabine or single agent imatinib. Primary end point was time to progression. No significant difference in median time to progression and median survival time was observed. Another phase II trial showed similar lack of efficacy of single agent imatinib in advanced pancreatic cancer (Gharibo 2005). A phase I/II study using the imatinib and gemcitabine combination for advanced pancreatic cancer was recently reported (McGartland et al 2006). This study has enrolled 17 patients so far with about $50 \%$ patients experiencing disease control (partial response or stable disease). Most common toxicities were neutropenia, fluid retention and fatigue. Dose escalation and further accrual continues.

\section{Sorafenib (BAY43-9006)}

Sorafenib is an oral, potent, dual action Raf protein kinase and VEGFR inhibitor with activity against PDGFR and c-Kit as well. The Ras oncogene is mutated in about $90 \%$ of pancreatic cancers, leading to constitutive activation of the Ras-Raf-MAPK signal transduction pathway. In preclinical studies sorafenib was shown to inhibit tumor angiogenesis and cell proliferation with broad-spectrum antitumor activity in colon, breast, and non-small-cell lung cancer xenograft models (Wilhelm et al 2004). Due to their compatibility in preclinical models and non-overlapping clinical toxicities, sorafenib was studied in advanced pancreatic cancer in combination with gemcitabine. A phase I study examined the combination in patients with advanced solid tumors, with an expanded cohort of patients with advanced pancreatic cancer (Siu et al 2006). This study enrolled 42 patients overall, with 23 patients in the extended pancreatic cancer cohort. Antitumor activity was observed in both groups, with 2 partial responses in ovarian cancer and 13 patients $(56.5 \%)$ in the pancreatic cohort achieving disease stabilization. Sorafenib and gemcitabine combination was well tolerated. Based on these results a multi-centre phase II trial of sorafenib and gemcitabine in advanced pancreatic cancer was conducted, the results of which were recently reported (Wallace et al 2007). Primary end-point was tumor response. No objective responses were observed in the first 17 patients enrolled on the study. The study closed to accrual as it did not meet primary end point.

\section{Cytoplasmic kinases Src}

Src kinases are a family of non-receptor protein tyrosine kinases, playing critical roles in cell division regulation, motility, adhesion, angiogenesis and survival (Summy and Gallick 2003). Src kinases are controlled by tyrosine kinase receptors (EGFR, PDGFR, fibroblast growth factor receptors), integrin receptors, G-protein coupled receptors, cytokine receptors, and steroid hormone receptors (Thomas and Brugge 1997). All Src family kinases contain a tyrosine kinase domain. On activation, Src signals to a variety of downstream effectors including Stat transcription factors. Constitutively activated variants of Src family kinases are capable of inducing malignant transformation. C-Src is frequently overexpressed and/or aberrantly activated in a variety of epithelial and non-epithelial cancers including colorectal, breast cancers, melanomas, ovarian cancer, lung cancer and pancreatic cancer. C-Src over-expression and elevated c-Src kinase activity was observed in pancreatic carcinoma cell lines. Src family kinases inhibitors are thus attractive options for future anti-cancer therapeutics.

\section{AZD0530}

AZD0530 is the earliest Src kinase TKI to undergo clinical testing in pancreatic cancer. AZD0530 has low anti-proliferative activity but inhibits tumor cell adhesion, migration and invasion. A clinical phase I/II trial combing AZD0530 with gemcitabine in advanced pancreatic cancer is currently being conducted.

\section{Dasatinib (BMS-354825)}

Dasatinib is an oral dual BCR/ABL and Src family tyrosine kinases inhibitor currently being tested in phase I settings in advanced pancreatic cancer patients.

\section{Future directions}

Tyrosine kinase inhibitors have presented a new lead in development of targeted therapies for treating pancreatic cancer. 
Even though preclinical evidence and phase I/II trials for activity of TKIs in pancreatic cancer have been encouraging, phase III clinical trials involving larger number of patients have been disappointing. Current challenges include improving patient selection, identifying effective combinations of various classes of agents, improving the predictive value of current preclinical models and better study designs (Mendelsohn and Baselga 2003).

1) Patient selection: The clinical activity of various agents so far has been observed in an unselected patient population. The heterogeneous nature of molecular abnormalities in pancreatic cancer would suggest that a targeted agent would be effective in only a relatively small subset of patients. The successful development of trastuzumab and imatinib in breast and sarcoma has been dependent on the ability to select patients with Her-2-neu overexpression and $c$-kit mutations. Therefore, it will be critical in the future trials to identify predictors of response to TKI and incorporate those predictors in patient selection for clinical trials.

2) The results of clinical trials in patients with stage IV pancreatic cancer indicate that a substantial number of patients (approximately 30\%) live less than 8 weeks. The likelihood that a therapeutic intervention will have a major impact within this short period of time is unlikely. Therefore, future consideration should be given to developing new agents in earlier stage disease or to applying more strict eligibility criteria for clinical trials in stage IV disease. Such criteria could include patients with better performance status, or patients with less baseline symptoms. The role of CA19-9 as a prognostic marker has been well established and could also be incorporated into the eligibility criteria of future trial (Hess et al 2008).

3) Drug combinations: Pancreatic cancer is dependent on multiple dysregulated signalling pathways. Therapeutic blockade of any single pathway may be overcome by activation of alternative receptors or pathways. Therefore, combining targeted agents is the next rational step in the clinical development of TKI. Unfortunately the rationale for combination therapy in the era of targeted agents is still largely based on empiricism and non overlapping toxicities. Given the large number of targets and available inhibitors, there is a need to develop a better understanding of the biology to support the selection of agents to be evaluated in combination therapy.

4) Preclinical models: Our knowledge about molecular abnormalities of pancreatic cancer is limited by the availability of preclinical models. Although many drug combinations have demonstrated additive and synergistic effects in xenograft models, few have shown significant clinical efficacy. There is a need for new preclinical models that would better predict the clinical activity of new agents.

5) Study design: With the large number of negative phase III trials, re-evaluation of the clinical trial paradigm has become necessary. Definitions of trial end-points used in current pilot phase II studies may need to be revisited (Mendelsohn and Baselga 2003). Possibly progression free survival or lack of change in tumor size may be more meaningful surrogates for survival than objective response. Randomized phase II studies or designs with multiple experimental arms may be necessary to choose the most effective and least toxic regimens for further testing in phase III trials.

\section{Conclusion}

The clinical development of tyrosines kinase inhibitors in pancreatic cancer is evolving rapidly as our understanding of tumor cell and receptor biology increases and the results of more clinical trials become available. Currently erlotinib is the sole tyrosine kinase inhibitor approved for use in pancreatic cancer. There is a need to better understand pancreatic tumor biology and molecular characterizations, improve patient selection and investigate appropriate combinations of targeted and chemotherapeutic agents to optimize treatment options.

\section{Disclosures}

The authors have conflicts of interest to disclose.

\section{References}

Arteaga CL. 2001. The epidermal growth factor receptor: from mutant oncogene in nonhuman cancers to therapeutic target in human neoplasia. $J$ Clin Oncol, 19(18 Suppl):32S-40S.

Baker CH, Solorzano CC, et al. 2001. Angiogenesis and cancer metastasis: antiangiogenic therapy of human pancreatic adenocarcinoma. Int J Clin Oncol, 6:59-65.

Bergers G, Song S, et al. 2003. Benefits of targeting both pericytes and endothelial cells in the tumor vasculature with kinase inhibitors. J Clin Invest, 111:1287-95.

Blaszkowsky LS, Earle C, et al. 2007. A phase II study of docetaxel in combination with ZD1839 (gefitinib) in previously treated patients with metastatic pancreatic cancer. J Clin Oncol, 2007 ASCO Ann Meeting Proc, 25(25 18S):15080.

Bramhall SR, Allum WH, et al. 1995. Treatment and survival in 13,560 patients with pancreatic cancer, and incidence of the disease, in the West Midlands: an epidemiological study. Br J Surg, 82:111-5.

Brand RE, Tempero MA. 1998. Pancreatic cancer. Curr Opin Oncol, 10:362-6.

Bruns CJ, Kohl G. 2003. Activity of ZD6474, a vascular endothelial growth factor receptor tyrosine kinase inhibitor (VEGFR(KDR)-TKI), ina model od ZD1839 (Iressa) resistance. Proc Am Soc Clin Oncol, 44:4880. 
Bruns CJ, Shrader M, et al. 2002. Effect of the vascular endothelial growth factor receptor-2 antibody DC101 plus gemcitabine on growth, metastasis and angiogenesis of human pancreatic cancer growing orthotopically in nude mice. Int J Cancer, 102:101-8.

Buchler P, Reber HA, et al. 2002. VEGF-RII influences the prognosis of pancreatic cancer. Ann Surg, 236:738-49; discussion 749.

Burris HA 3rd, Moore MJ, et al. 1997. Improvements in survival and clinical benefit with gemcitabine as first-line therapy for patients with advanced pancreas cancer: a randomized trial. J Clin Oncol, 15:2403-13

Chen J, Rocken C, et al. 2006. The tyrosine kinase inhibitor imatinib fails to inhibit pancreatic cancer progression. Cancer Lett, 233:328-37.

Cherrington JM, Strawn LM, et al. 2000. New paradigms for the treatment of cancer: the role of anti-angiogenesis agents. Adv Cancer Res, 79:1-38.

Ciardiello F, Caputo R, et al. 2003. Antitumor effects of ZD6474, a small molecule vascular endothelial growth factor receptor tyrosine kinase inhibitor, with additional activity against epidermal growth factor receptor tyrosine kinase. Clin Cancer Res, 9:1546-56.

Conrad C, Ischenko I, et al. 2007. Antiangiogenic and antitumor activity of a novel vascular endothelial growth factor receptor-2 tyrosine kinase inhibitor ZD6474 in a metastatic human pancreatic tumor model. Anticancer Drugs, 18:569-79.

Dong M, Nio Y, et al. 1998. Epidermal growth factor and its receptor as prognostic indicators in Chinese patients with pancreatic cancer. Anticancer Res, 18:4613-9.

Dvorak HF. 2002. Vascular permeability factor/vascular endothelial growth factor: a critical cytokine in tumor angiogenesis and a potential target for diagnosis and therapy. J Clin Oncol, 20:4368-80.

Ebert M, Yokoyama M, et al. 1995. Induction of platelet-derived growth factor $\mathrm{A}$ and $\mathrm{B}$ chains and over-expression of their receptors in human pancreatic cancer. Int $J$ Cancer, 62:529-35.

El-Rayes BF, Philip PA. 2003. A review of systemic therapy for advanced pancreatic cancer. Clin Adv Hematol Oncol, 1:430-4.

Epelbaum R, Gluzman SJ, et al. 2007. Erlotinib as a single-agent therapy in patients with advanced pancreatic cancer. ASCO 2007 Gastrointestinal Cancers Symposium. Abstr 202.

Ferrara N. 1999. Molecular and biological properties of vascular endothelial growth factor. $J$ Mol Med, 77:527-43.

Folkman J. 1971. Tumor angiogenesis: therapeutic implications. N Engl J Med, 285:1182-6.

Gharibo MM, Juvidian P, et al. 2005. Phase II trial of Gleevec (Imatinib mesylate) in patients with metastatic unresectable pancreatic cancer. J Clin Oncol, 2005 ASCO Ann Meeting Proc, 23(16S):4183.

Heldin CH, Westermark B. 1999. Mechanism of action and in vivo role of platelet-derived growth factor. Physiol Rev, 79:1283-316.

Hess V, Glimelius B, et al. 2008. CA19-9 tumour-marker response to chemotherapy in patients with advanced pancreatic cancer enrolled in a randomised controlled trial. Lancet Oncol, 9:132-8

Hicklin DJ, Ellis LM. 2005. Role of the vascular endothelial growth factor pathway in tumor growth and angiogenesis. J Clin Oncol, 23:1011-27.

Hubbard SR. 2006. EGF receptor activation: push comes to shove. Cell, 125:1029-31

Hwang RF, Yokoi K, et al. 2003. Inhibition of platelet-derived growth factor receptor phosphorylation by STI571 (Gleevec) reduces growth and metastasis of human pancreatic carcinoma in an orthotopic nude mouse model. Clin Cancer Res, 9:6534-44.

Iannitti D, Dipetrillo T, et al. 2005. Erlotinib and chemoradiation followed by maintenance erlotinib for locally advanced pancreatic cancer: a phase I study. Am J Clin Oncol, 28:570-5.

Ikeda N, Adachi M, et al. 1999. Prognostic significance of angiogenesis in human pancreatic cancer. Br J Cancer, 79:1553-63.

Inai T, Mancuso M, et al. 2004. Inhibition of vascular endothelial growth factor (VEGF) signaling in cancer causes loss of endothelial fenestrations, regression of tumor vessels, and appearance of basement membrane ghosts. Am J Pathol, 165:35-52.
Itakura J, Ishiwata T, et al. 2000. Concomitant over-expression of vascular endothelial growth factor and its receptors in pancreatic cancer. Int $J$ Cancer, 85:27-34.

Jemal A, Siegel R, et al. 2007. Cancer statistics, 2007. CA Cancer J Clin, 57:43-66.

Kleespies A, Jauch KW, et al. 2006. Tyrosine kinase inhibitors and gemcitabine: new treatment options in pancreatic cancer? Drug Resist Updat, 9:1-18.

Kuo T, Kaiser FA, et al. 2006. A phase I study of the VEGF receptor tyrosine kinase inhibitor vatalanib (PTK787/ZK 222584) and gemcitabine in patients with advanced pancreatic cancer. J Clin Oncol, 2006 ASCO Ann Meeting Proc, 24(18S):4122.

Lemoine NR, Hughes CM, et al. 1992. The epidermal growth factor receptor in human pancreatic cancer. J Pathol, 166:7-12.

Levitzki A. 1999. Protein tyrosine kinase inhibitors as novel therapeutic agents. Pharmacol Ther, 82:231-9.

Levitzki A, Gazit A. 1995. Tyrosine kinase inhibition: an approach to drug development. Science, 267:1782-8.

Li J, Kleeff J, et al. 2004. Gefitinib ('Iressa', ZD1839), a selective epidermal growth factor receptor tyrosine kinase inhibitor, inhibits pancreatic cancer cell growth, invasion, and colony formation. Int $J$ Oncol, 25:203-10.

Louvet C, Labianca R, et al. 2005. Gemcitabine in combination with oxaliplatin compared with gemcitabine alone in locally advanced or metastatic pancreatic cancer: results of a GERCOR and GISCAD phase III trial. J Clin Oncol, 23:3509-16.

Luo J, Guo P, et al. 2001. Pancreatic cancer cell-derived vascular endothelial growth factor is biologically active in vitro and enhances tumorigenicity in vivo. Int $J$ Cancer, 92:361-9.

McGartland LP, Rademaker A, et al. 2006. Phase I/II study of imatinib mesylate and gemcitabine for advanced pancreas cancer. ASCO 2006 Gastrointentinal Cancers Symposium, Abstr 132.

Mendelsohn J, Baselga J. 2003. Status of epidermal growth factor receptor antagonists in the biology and treatment of cancer. J Clin Oncol, 21:2787-99.

Mendelsohn J, Baselga J. 2006. Epidermal growth factor receptor targeting in cancer. Semin Oncol, 33:369-85.

Moore MJ, Goldstein D, et al. 2007. Erlotinib plus gemcitabine compared with gemcitabine alone in patients with advanced pancreatic cancer: a phase III trial of the National Cancer Institute of Canada Clinical Trials Group. J Clin Oncol, 25:1960-6.

Niedergethmann M, Hildenbrand R, et al. 2002. High expression of vascular endothelial growth factor predicts early recurrence and poor prognosis after curative resection for ductal adenocarcinoma of the pancreas. Pancreas, 25:122-9.

Oettle H, Post S, et al. 2007. Adjuvant chemotherapy with gemcitabine vs observation in patients undergoing curative-intent resection of pancreatic cancer: a randomized controlled trial. JAMA, 297:267-77.

Olayioye MA, Neve RM, et al. 2000. The ErbB signaling network: receptor heterodimerization in development and cancer. Embo J, 19:3159-67.

Ostman A. 2004. PDGF receptors-mediators of autocrine tumor growth and regulators of tumor vasculature and stroma. Cytokine Growth Factor Rev, 15:275-86.

Papageorgio C, Perry MC. 2007. Epidermal growth factor receptor-targeted therapy for pancreatic cancer. Cancer Invest, 25:647-57.

Philip P, Benedetti J, et al. 2007. Phase III study of gemcitabine plus cetuximab versus gemcitabine in patients woth locally advanced or metastatic pancreatic adenocarcinoma: SWOG S0205 study. J Clin Oncol, 25:965s.

Pietras K, Rubin K, et al. 2002. Inhibition of PDGF receptor signaling in tumor stroma enhances antitumor effect of chemotherapy. Cancer Res, 62:5476-84.

Regine WF, Abrams RA. 2006. Adjuvant therapy for pancreatic cancer: current status, future directions. Semin Oncol, 33(Suppl 11):S10-3.

Ritter CA, Arteaga CL. 2003. The epidermal growth factor receptor-tyrosine kinase: a promising therapeutic target in solid tumors. Semin Oncol, 30(Suppl 1):3-11. 
Rocha Lima CM, Green MR, et al. 2004. Irinotecan plus gemcitabine results in no survival advantage compared with gemcitabine monotherapy in patients with locally advanced or metastatic pancreatic cancer despite increased tumor response rate. J Clin Oncol, 22:3776-83.

Rugo HS, Herbst RS, et al. 2005. Phase I trial of the oral antiangiogenesis agent AG-013736 in patients with advanced solid tumors: pharmacokinetic and clinical results. J Clin Oncol, 23:5474-83.

Safran H, Miner K et al. 2006. GW572016, gemcitabine and GW572016, gemcitabine, oxaliplatin, a two-stage, phase I study for advanced pancreaticobiliary cancer. J Clin Oncol, 2006 ASCO Ann Meeting Proc, 24(18S):4002.

Schlessinger J. 2000. Cell signaling by receptor tyrosine kinases. Cell, 103:211-25.

Siu LL, Awada A, et al. 2006. Phase I trial of sorafenib and gemcitabine in advanced solid tumors with an expanded cohort in advanced pancreatic cancer. Clin Cancer Res, 12:144-51.

Spano JP, Kim S, et al. 2006. A phase I study of Axitinib (AG-013736), a potent inhibitor of VEGFRs, in combination with gemcitabine (GEM) in patients (pts) with advanced pancreatic cancer. J Clin Oncol, 2006 ASCO Ann Meeting Proc, 24(18S):13092.

Spano JP, Chodkiewicz C, et al. 2008. Efficacy of gemcitabine plus axitinib compared with gemcitabine alone in patients with advanced pancreatic cancer: an open-label randomised phase II study. Lancet. XXXX.

Summy JM, Gallick GE. 2003. Src family kinases in tumor progression and metastasis. Cancer Metastasis Rev, 22:337-58.

Thomas SM, Brugge JS. 1997. Cellular functions regulated by Src family kinases. Annu Rev Cell Dev Biol, 13:513-609.
Vervenne W, Humblet Y, et al. 2008. A randomized, double-blind, placebo (P) controlled, multicenter phase III trial to evaluate the efficacy and safety of adding bevacizumab (B) to erlotinib (E) and gemcitabine $(\mathrm{G})$ in patients (pts) with metastatic pancreatic cancer. J Clin Oncol, 2008 ASCO Annual Meeting, 26(Suppl):abstr 4507).

Wallace JA, Nattam S, et al. 2007. Sorafenib plus gemcitabine for advanced pancreatic cancer : A phase II trial of the University of Chicago Phase II Consortium. Journal of Clinical Oncol, 2007 ASCO Ann Meeting Proc, 25(18S) 4608.

Wilhelm SM, Carter C, et al. 2004. BAY 43-9006 exhibits broad spectrum oral antitumor activity and targets the RAF/MEK/ERK pathway and receptor tyrosine kinases involved in tumor progression and angiogenesis. Cancer Res, 64:7099-109.

Wood JM, Bold G, et al. 2000. PTK787/ZK 222584, a novel and potent inhibitor of vascular endothelial growth factor receptor tyrosine kinases, impairs vascular endothelial growth factor-induced responses and tumor growth after oral administration. Cancer Res, 60:2178-89.

Yamanaka Y, Friess H, et al. 1993. Coexpression of epidermal growth factor receptor and ligands in human pancreatic cancer is associated with enhanced tumor aggressiveness. Anticancer Res, 13:565-9.

Yarden Y, Sliwkowski MX. 2001. Untangling the ErbB signalling network. Nat Rev Mol Cell Biol, 2:127-37.

Yokoi K, Sasaki T, et al. 2005. Simultaneous inhibition of EGFR, VEGFR, and platelet-derived growth factor receptor signaling combined with gemcitabine produces therapy of human pancreatic carcinoma and prolongs survival in an orthotopic nude mouse model. Cancer Res, 65:10371-80 
